\title{
PERIPHERAL PRIMITIVE NEUROECTODERMAL TUMOUR OF THE PLEURA IN A YOUNG ADULT- A CASE REPORT
}

Arpita Mahapatra Thamba', Kriti Malhotra²

${ }^{1}$ Associate Professor, Department of Radiodiagnosis, K. J. Somaiya Medical College.

${ }^{2}$ Senior Resident, Department of Radiodiagnosis, K. J. Somaiya Medical College.

HOW TO CITE THIS ARTICLE: Thamba AM, Malhotra K. Peripheral primitive neuroectodermal tumour of the pleura in a young adult- a case report. J. Evolution Med. Dent. Sci. 2017;6(54):4107-4109, DOI: 10.14260/Jemds/2017/887

\section{PRESENTATION OF CASE}

A 25-year-old male presented with history of respiratory distress, acute onset from 2 days. On plain chest radiograph, there was obliteration of left cardiophrenic angle with homogenous opacity in left lower and middle zone with obliteration of left cardiac and diaphragmatic silhouette suggestive of massive pleural effusion. Next, an ultrasound of chest was done for guiding intercostal tube for drainage of pleural effusion where upon hyperechoic pleural based lesions were seen. The chest drain inserted drained.

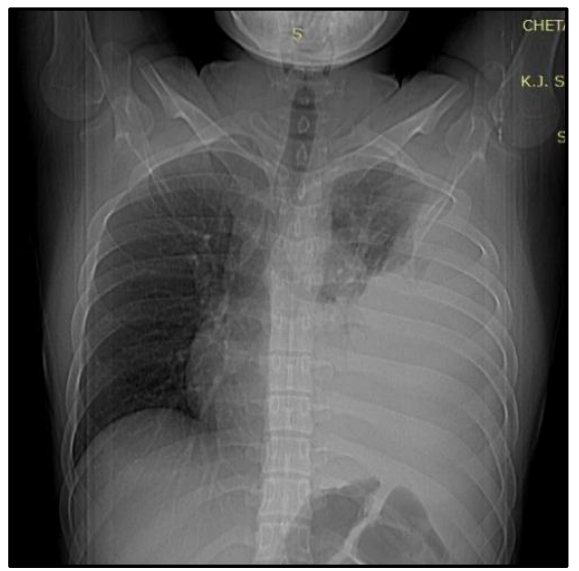

Figure 1. Gross Right Pleural Effusion with Partial Collapse of underlying Lung Field. No obvious Bone Destruction is Seen

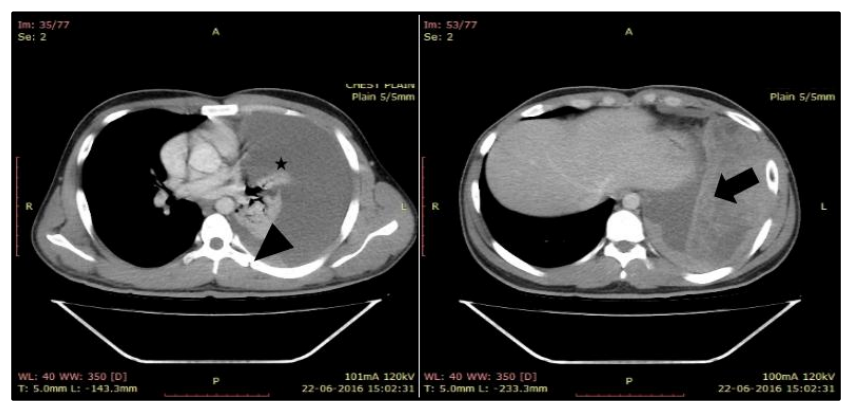

Figure 2. Contrast Enhanced Axial CT Images show Large Left Pleural Effusion (Asterisk) with Heterogeneously Hyperdense Large Area in Left Pleural Cavity (Arrow). Also noted similar Pleural Based Lesion (Arrow Head). Overlying Ribs and Thoracic Wall appears Normal

Financial or Other, Competing Interest: None.

Submission 24-04-2017, Peer Review 23-06-2017,

Acceptance 29-06-2017, Published 06-07-2017.

Corresponding Author:

Dr. Arpita Mahapatra Thamba,

\#17, Bharat Tirtha Society,

V. N. Purav Marg,

Chembur, Mumbai-400071

E-mail: arpita_rad@yahoo.com

DOI: $10.14260 /$ jemds $/ 2017 / 887$

\section{(c) (i) $($ )}

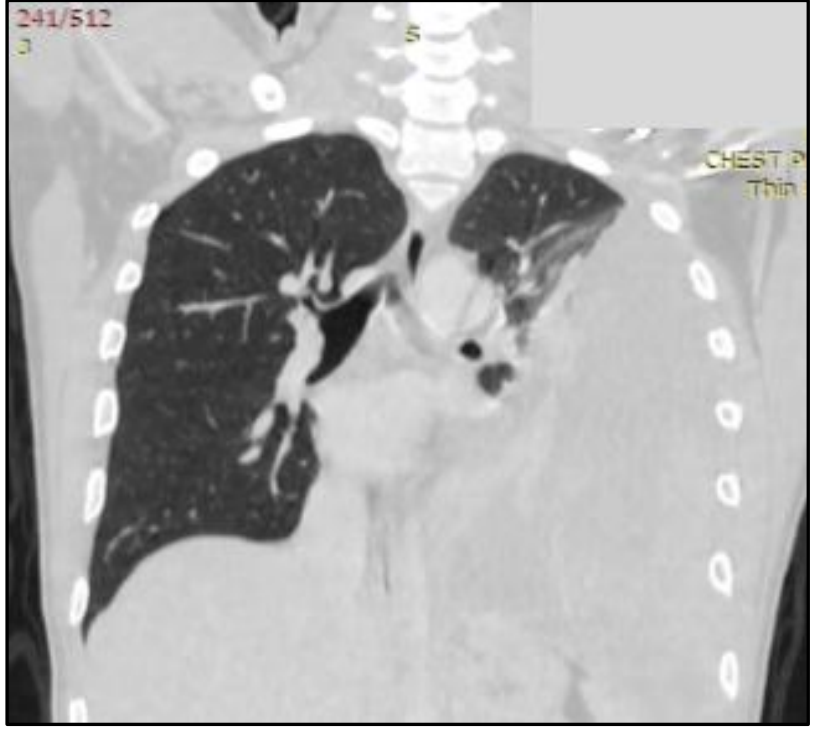

Figure 3. Coronal CT Images show Passive Collapse of Left Lung Field

Haemorrhagic fluid with thick clots. The fluid cytology did not reveal any malignant cells. The Mantoux was negative with no history of contact Koch's. He was further investigated with Contrast Enhanced Computed Tomography (CECT) of the chest in view of haemorrhagic fluid and pleural based lesions to rule out malignant or traumatic aetiology. CECT chest revealed a large left pleural effusion with a large heterogeneously hyperdense lesion within the pleural cavity with no obvious enhancement. Similar density lesions with subtle enhancement were seen along the pleura (Fig. 2). There was no evidence of involvement of ribs or chest wall. The ipsilateral lung field showed passive collapse (Fig. 3). Patient was taken up for thoracotomy and a large haemorrhagic clot with pleural effusion was drained. The specimen received showed greyish friable material on the clot pieces. A followup CECT chest (Fig. 4) was done which showed significant reduction of pleural effusion. The large heterogeneous lesion was absent on this scan; however, the pleural based lesions still persisted. The material was sent for histopathological examination. The microscopic diagnosis was malignant, small round cell tumour, hemithorax. Further immunohistochemistry study was carried out for a definitive diagnosis. It was consistent with PNET being positive for vimentin, CD99 and FLI-1 (friend leukaemia integration-1) and negative for desmin, mesothelin and cytokeratin. Now after three months the patient being on multidrug chemotherapy, the tumour has shown significant regression in followup computed tomography (Fig. 5). 

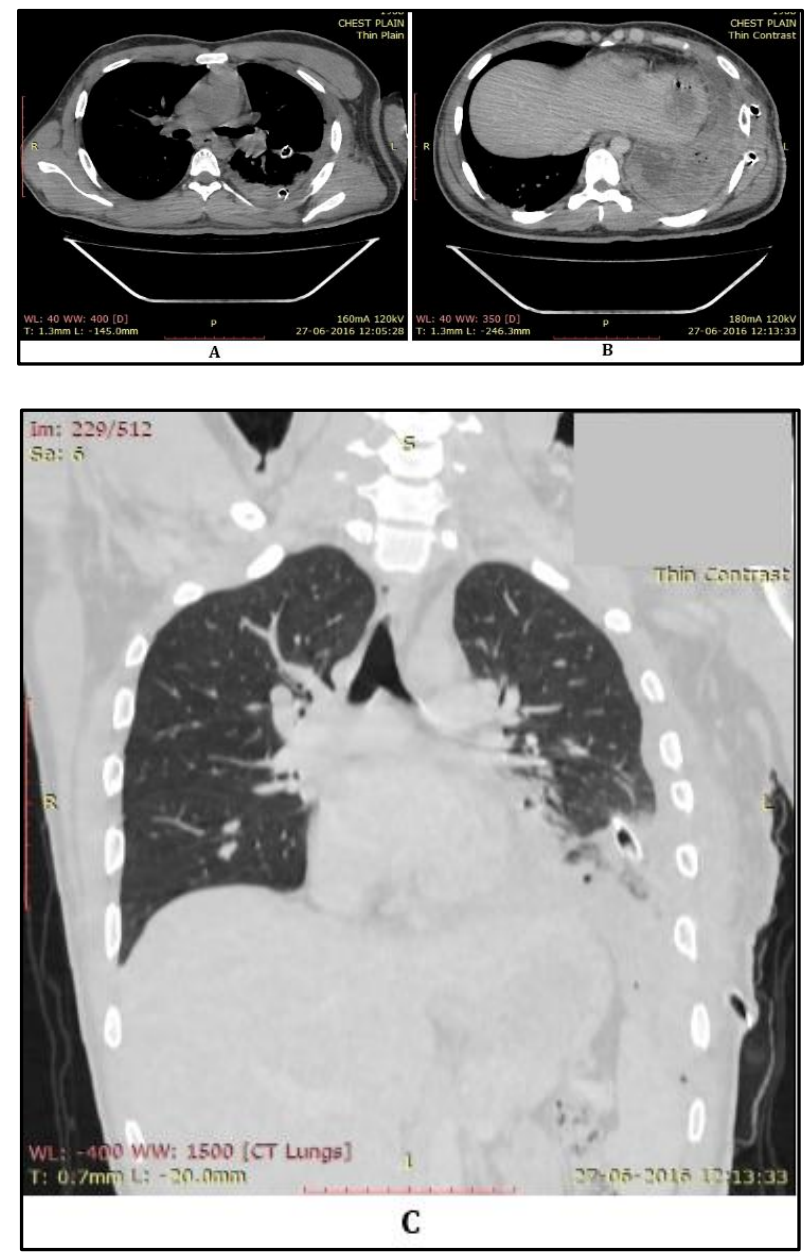

Figure 4. Followup CECT Thorax after Thoracotomy reveals (A) significant resolution of Left Pleural Effusion with Drainage Tubes in situ; (B) Persistence of the PleuralBased Lesions; (C) Significant Re-Expansion of Left Lung Field

\section{DIFFERENTIAL DIAGNOSIS}

Primitive Neuroectodermal Tumours (PNET) are a group of highly malignant tumours composed of small round cells of neuroectodermal origin that affect soft tissue and bone.(1) The term PNET was coined by Hart and Earle(2) in 1973 for predominantly undifferentiated tumours of the cerebellum that did not match the diagnostic criteria for neuroblastoma, ependymoblastoma, medulloblastoma or pineal parenchymal tumours. However, they were first described by Arthur Purdy Stout in 1918.(3) These tumours may originate in central nervous system or other tissues peripherally. The peripheral PNETs are typically seen in soft tissues of chest wall, paraspinal region, soft tissue of head and neck and genitourinary tract. PNETs located in thoracopulmonary region are often referred to as Askin's tumour.(4) We report a case of pPNET of the pleura in a young adult and discuss the diagnostic and clinicopathological significance of the lesion.

\section{PATHOLOGICAL DISCUSSION}

On CT scans PNETs appear as ill-defined, hypodense, heterogeneous masses with or without invasion of surrounding tissue. These lesions have ill-defined margins with irregular shapes. On contrast enhancement, majority of the lesions show heterogeneous enhancement with areas of necrosis. Unlike our case, which shows no obvious contrast enhancement. Calcification is rarely seen in these tumours.(5)
Ipsilateral hilar and mediastinal adenopathy and pneumothorax are other rare findings.(6)

MR imaging show heterogeneous lesions with signal intensity isointense to spinal cord on $\mathrm{T} 1$ weighted images and hyperintense on $\mathrm{T} 2$ weighted and proton density images. Direct invasion into surrounding structures can be beautifully demonstrated on MR imaging. Post contrast imaging shows heterogeneous contrast uptake and shows well demarcated borders of the tumour.(7)

Distant metastasis has been known to spread to lungs, mediastina and retroperitoneal nodes, bones, diaphragm, liver, adrenals, sympathetic nerve chain and brain.(8)(9)

\section{DISCUSSION OF MANAGEMENT}

Peripheral Primitive Neuroectodermal Tumours (PNETs) are a group of small round cell malignancies with neural crest origin that arise outside central and sympathetic nervous system.(10) It is a rare disease in children as well as in adults. These are classified as a part of Ewing's sarcoma family of tumours. Ewing's and pPNETs may have different manifestations, but have similar histological and immunohistochemical characteristics. Both includes genetic translocations of chromosomes 11 and 22 giving rise to Ewing's Sarcoma Gene (EWS)/ Friend Leukaemia virus Integration-1 gene (FLI-1).(11) Homer Wright rosettes are a marker for pPNET. Peripheral PNETs typically co-express CD (glycoprotein MIC2) and vimentin. Other markers include neuron-specific enolase, Leu-7 (later termed CD57) and S100.(12)

Patients usually present with chest pain and respiratory distress. Review of radiological findings showed that most cases of pleural PNETs similar to ours presents with massive pleural effusion, occasionally haemorrhagic or loculated. Few patients may show pulmonary involvement. Compression of underlying lung is often difficult to differentiate from parenchymal involvement.(4) Aggressive masses often invade the chest wall, pericardium and vertebrae with spinal extension and ribs.

Multidisciplinary approach is used for treatment of Askin's tumour involving surgery, radiotherapy and chemotherapy. Neoadjuvant chemotherapy is the first choice of treatment followed by surgical resection. Chemotherapy includes vincristine, doxorubicin, cyclophosphamide alternating with etoposide and IFM.(13)

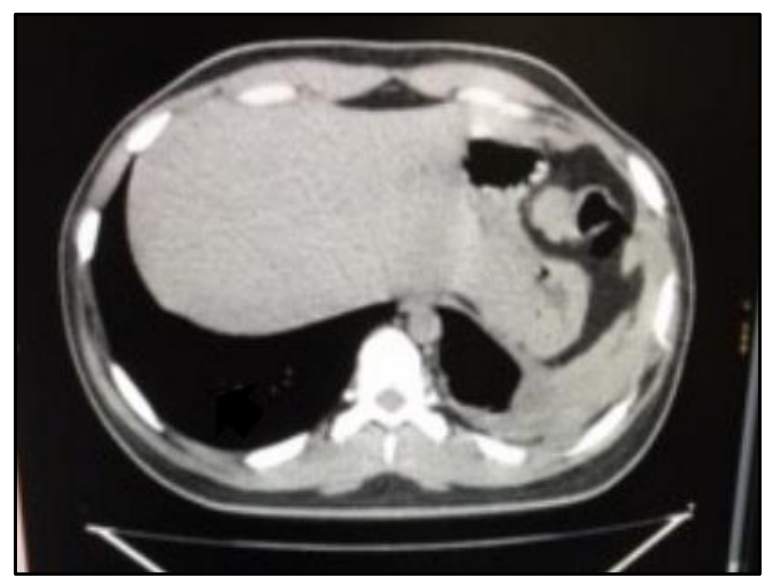

Figure 5. Significant Resolution of Pleural Based Lesions seen after three months of Chemotherapy with Complete Resolution of Left Pleural Effusion. Note the Presence of Pleural Thickening noted on Left Side (Block Arrow) 


\section{CONCLUSION}

Although Askin's tumours are difficult to distinguish from other sarcomas, only on imaging basis the differential of peripheral PNETs is to be considered when a heterogeneous mass is seen in thoracopulmonary region, especially with local invasion in young adults. The diagnosis is dependent upon histopathology and immunohistochemistry. Early detection plays a crucial role in proper treatment.

\section{FINAL DIAGNOSIS}

Peripheral Primitive Neuroectodermal Tumour of the Pleura.

\section{REFERENCES}

[1] Virani MJ, Jain S. Primary intraspinal primitive neuroectodermal tumor (PNET): a rare occurrence. Neurol India 2002;50(1):75-80.

[2] Hart MN, Earle KM. Primitive neuroectodermal tumors of the brain in children. Cancer 1973;32(4):890-7.

[3] Stout AP. A tumor of the ulnar nerve. Proc NY Pathol Soc 1918;18(2):2-12.

[4] Winer-Muram HT, Kauffman WM, Gronemeyer SA, et al. Primitive neuroectodermal tumors of the chest wall (Askin tumors): CT and MR findings. AJR Am J Roentgenol 1993;161(2):265-8.

[5] Xia T, Guan Y, Chen Y, et al. Askin tumor: CT and FDG$\mathrm{PET} / \mathrm{CT}$ imaging findings and follow-up. Medicine 2014;93(6):e42.
[6] Fink IJ, Kurtz DW, Cazenave L, et al. Malignant thoracopulmonary small-cell ("Askin") tumor. AJR Am J Roentgenol 1985;145(3):517-20.

[7] Faubert C, Inniger R. MRI and pathological findings in two cases of askin tumors. Neuroradiology 1991;33(3):277-81.

[8] Christiansen S, Semik M, Dockhorn-Dworniczak B, et al. Diagnosis, treatment and outcome of patients with askin-tumors. Thorac Cardiovasc Surg 2000;48(5):311-5.

[9] Fink M, Salisbury J, Gishen P. Askin tumor: three case histories and a review of the literature. European Journal of Radiology 1992;14(3):178-80.

[10] Gong J, Zhang Y, Zuo M, et al. Imaging findings of abdominal peripheral primitive neuroectodermal tumor: report of four cases with pathological correlation. Clin Imaging 2009;33(3):196-9.

[11] Rorie CJ, Thomas VD, Chen P, et al. The Ews/Fli-1 fusion gene switches the differentiation program of neuroblastomas to ewing sarcoma/peripheral primitive neuroectodermal tumors. Cancer Res 2004;64(4):1266-77.

[12] Pinto A, Dickman P, Parham D. Pathobiologic markers of the ewing sarcoma family of tumors: state of the art and prediction of behaviour. Sarcoma 2011;2011:856190.

[13] Dou X, Yan H, Wang R. Treatment of an askin tumor: a case report and review of the literature. Oncol Lett 2013;6(4):985-9. 